\title{
Horizons philosophiques
}

\section{Liminaire}

\section{Michèle Émond}

Volume 15, numéro 1, automne 2004

En quête du sujet

URI : https://id.erudit.org/iderudit/801272ar

DOI : https://doi.org/10.7202/801272ar

Aller au sommaire du numéro

Éditeur(s)

Collège Édouard-Montpetit

ISSN

1181-9227 (imprimé)

1920-2954 (numérique)

Découvrir la revue

Citer ce document

Émond, M. (2004). Liminaire. Horizons philosophiques, 15(1), I-IV.

https://doi.org/10.7202/801272ar

Ce document est protégé par la loi sur le droit d'auteur. L'utilisation des services d'Érudit (y compris la reproduction) est assujettie à sa politique d'utilisation que vous pouvez consulter en ligne.

https://apropos.erudit.org/fr/usagers/politique-dutilisation/
Cet article est diffusé et préservé par Érudit.

Érudit est un consortium interuniversitaire sans but lucratif composé de l'Université de Montréal, l'Université Laval et l'Université du Québec à Montréal. Il a pour mission la promotion et la valorisation de la recherche. https://www.erudit.org/fr/ 


\section{Liminaire}

Le sujet a été passablement malmené au $20^{\circ}$ siècle. À peine remis des prétentions de la psychanalyse à restreindre le champ de sa conscience et de son libre arbitre, il lui a fallu recomposer son histoire en tenant compte des grands changements sociaux et culturels découlant de l'extension de la modernité. Les épreuves et traumatismes collectifs subis lors des grands conflits politiques ont suscité un intérêt grandissant pour le récit des répercussions de ces drames dans la vie des individus. Lors de ces expériences souvent partagées, banales, et parfois extrêmes, l'individu a dû redéfinir son identité, tant le sens commun qui assurait la cohérence de son histoire n'allait plus de soi. Les périodes de rupture avec le monde habituel ont donc créé les conditions d'une possible crise d'identité. Ainsi, plus les mondes sociaux et les modèles culturels s'ouvrent et se différencient, plus l'individu est déstabilisé et condamné à se redéfinir comme sujet.

Les témoignages recueillis auprès de migrants, de rescapés des camps, des derniers habitants de régions en voie de modernisation ont exprimé différentes dimensions de cette rupture et manifesté une quête de reconstruction d'identité, à la fois individuelle et collective. La visée politique et éducative de ces témoignages est omniprésente dans le cas des récits de la Shoah par exemple, ou de ceux des victimes de l'apartheid. Des visées plus psychologiques ou sociales ont accompagné l'engouement pour la méthode de recueil de récits de vie, tant au plan de la recherche que de la démarche personnelle d'exploration de la trajectoire d'un individu dans son milieu et son époque. La richesse des témoignages ou autobiographies recueillis en histoire orale a aussi fait émerger un acteur nouveau, l'individu singulier, dont la notoriété l'autorisant à prendre la parole est basée surtout sur sa participation à des événements ordinaires ou à son statut de représentant ou de porte-parole d'un groupe. Pour certains récits, comme celui de Primo Lévi, la valeur individuelle du témoignage ouvre à la reconnaissance de l'appartenance au groupe le plus large qu'on puisse imaginer : l'humanité.

C'est à la narration de cet individu singulier que se sont intéressées la philosophie et les sciences de l'homme dans leurs 
travaux autour du travail de construction de l'identité narrative. C'est un champ d'analyse des récits, romans, contes où s'exprimerait le travail de gestion de l'identité qui consiste à créer des histoires cohérentes à partir d'expériences de vie parfois chaotiques et sans beaucoup de sens. Des questions comme celles soulevées par Paul Ricœur dans Soi-même comme un autre ${ }^{1}$ ont élargi la compréhension des histoires que l'on raconte à son propre sujet où la personne est comprise comme narrateur et personnage d'une histoire qui tente de s'inscrire dans le temps. C'est en se racontant que l'on aurait accès à la temporalité de l'existence et seul un être capable de rassembler sa vie sous la forme d'un récit pourrait accéder à une identité éthique.

Le récit de soi a aussi intéressé tout un courant de recherches et de pensée qui s'est même ramifié par la suite. La pratique des récits de vie en sociologie des professions, celle autour des histoires de vie en formation ou les auto-analyses, telle celle produite par Pierre Bourdieu seraient dans le prolongement et le raffinement de ces tentatives de rendre compte d'une vie, d'un individu. Pourrait-on même aller jusqu'à associer la vogue actuelle pour l'auto-fiction à ce désir profond de se construire comme sujet par le récit?

Le texte de Michel Legrand qui ouvre ce numéro offre un aperçu de la contribution originale de cet auteur à l'analyse biographique. Situant l'engouement pour l'histoire de vie dans le prolongement de l'idéologie individualiste qui nous invite à devenir le sujet de notre récit, si ce n'est de notre vie, l'auteur situe sa démarche dans une posture constructiviste "modérée» qui ne serait pas volonté de cohérence à tout prix, mais qui laisserait une place à l'absurde, au non-sens. Développant cette idée à l'aide du thème de l'événement comme catégorie biographique signifiant le hasard, mais faisant intervenir le sujet, ses choix et finalement le destin, l'auteur nous entraîne dans une relecture de la trajectoire de vie de Flaubert, telle que tracée par Sartre dans L'Idiot de la famille. Ce que Michel Legrand a deviné dans l'explication de Sartre concernant la transformation d'une catastrophe en destin, c'est un espace "d'entre-deux" où un sujet peut se mouvoir : un espace entre la contrainte et la liberté.

Didier Moreau s'interroge sur l'essor des histoires de vie en lien 
avec l'ouverture d'une possibilité d'orientation nouvelle du "soi" autorisée par la fin des cadres classiques de recueil de soi, dans le contexte actuel de la post-historicité. Le détour par les thématiques antiques du peregrinans et du proficiens servira à éclairer la synthèse qu'en fera Hegel et son interprétation par Heidegger comme fin de la recherche d'un achèvement. II montre ensuite comment l'expérience de la post-historicité rentre alors dans la voie d'une hermétique du pro-jet qui détermine la présence comme Ereignis. L'auteur conclut que l'injonction à entrer dans la posthistoricité est peut-être une "chance", celle par laquelle la démarche de se recueillir dans I'histoire de vie devient aussi affirmation, acceptation de notre mort, reconnaissance des autres qui nous ont précédés et projet de transmettre notre expérience à nos successeurs.

En éclairant les présupposés d'un débat connu entre Gadamer et Derrida qui illustre comment le langage est devenu le lieu où s'éprouvent les limites de la subjectivité, François Doyon «montre, à partir de l'étude des positions des deux philosophes, mais aussi de leurs prédécesseurs reconnus, comme Heidegger, ou trop peu souvent cités comme Husserl, mais surtout Nietzsche, dans quelle mesure la critique que Derrida adresse à l'herméneutique peut être juste, ce qui (l')amènera à voir comment la recherche de sens par la pensée peut être étouffée par la finitude du langage humain».

Henri Bah s'inspire ici de la pensée bergsonienne pour poser le problème du sens de la vie. Pour Bergson, on le sait, la vie révèle qu'elle est durée, étoffe dont est faite toute réalité. La durée constitue le cœur même de la vie. C'est à travers l'expérience des âmes héroïques ou mystiques que Bergson a cru déceler la source de cette durée qui lui fait dire que le sens de la vie, c'est l'amour. Le héros bergsonien ne serait donc pas un simple modèle, mais sa vie exprimerait le sens même de l'être de la vie dans sa totalité. Cette contribution rappelle que plusieurs penseurs proposent encore une "transcendance horizontale" qui relierait les êtres humains entre eux à partir des grands récits chrétiens ou de ceux des héros ordinaires œuvrant à élever l'univers vers l'humanité.

C'est à une réflexion sur sa vie et sa mise en récit que Luis Gomez nous convie en faisant l'analyse de son parcours de recherche centré sur l'histoire de vie comme espace de construction 
identitaire. Des liens pourront être tissés entre l'analyse d'un événement catastrophique proposée par Michel Legrand et celle livrée par l'auteur qui déconstruit-reconstruit son histoire à partir de l'annonce d'une catastrophe dans sa vie personnelle. S'entend aussi l'écho des recherches sociologiques ${ }^{2}$ menées sur les malades chroniques et sur leur travail introspectif de mise en cohérence de leur parcours et de leur identité à partir du souci d'exprimer leur vulnérabilité et de la faire entendre à l'autre.

Pierre-Luc Lortie situe lui aussi son survol des mutations actuelles du sacré dans le contexte particulier d'une société où la construction de cohérence et de sens est devenue une entreprise individuelle. À partir de l'analyse des "communautés de sensation" que sont les raves, l'auteur dégage quelques pistes qui lui permettent de situer le phénomène rave comme une réponse religieuse à une quête de sens. Qu'on les comprenne comme un lieu d'expression d'un nouveau tribalisme ou comme une forme spontanée de rituel sauvage, hédoniste, les raves «souligneraient la nécessité symbolique du regroupement et du partage de moments communs d'intensité".

En se penchant sur la dimension esthétique de l'existence humaine, Bruno Demers a voulu développer sa réflexion sur la question des aspects sensibles qui marquent le rapport de l'être humain au monde. Adoptant une approche qui s'appuie sur des concepts tirés de la sociologie, l'anthropologie et la philosophie, l'auteur explore l'idée marxienne selon laquelle l'homme produit son existence selon les lois de la beauté, qui seront ici cernées en recourant aux concepts de style de vie et de normativité. Cet examen amènera à questionner la place de la norme en matière esthétique dans une société où l'homme est invité à définir ses propres critères de beauté.

Michèle Émond

Sociologie, collège Édouard-Montpetit

1. Paul Ricœur, Soi-même comme un autre, Paris, Seuil, 1990.

2. On trouvera un aperçu de ces recherches dans Michaël Voegtli, «Du Jeu dans le $\mathrm{Je}$ : ruptures biographiques et travail de mise en cohérence", Lien social et Politiques - RIAC, 51, Engagement social et politique dans le parcours de vie, Printemps 2004, p. 145-158. 